\title{
REDES DE APOIO À PESSOA COM DEFICIÊNCIA FÍSICA
}

\section{SUPPORT NETWORK FOR PEOPLE WITH DISABILITIES}

\section{RED DE APOYO PARA LAS PERSONAS CON DISCAPACIDAD}

\author{
Daniely Monteiro Cruz ${ }^{*}$ \\ Luis Ramon SOUSA do NASCIMENTO ** \\ Denise Maria Guerreiro Vieira Da Silva ${ }^{* * *}$ \\ SORAIA DORNELlES SCHOELLER ${ }^{* * *}$
}

\begin{abstract}
RESUMO
Este estudo versa sobre a rede de apoio à pessoa com deficiência física e de que forma esse apoio é prestado. Seu objetivo foi conhecer as redes de apoio à pessoa com deficiência física. Resulta de uma pesquisa descritiva com abordagem qualitativa, com base em entrevistas semi-estruturadas com 13 pessoas com deficiência física, sendo seis com amputação e sete com lesão medular atendidas em um centro de reabilitação. Foi utilizada análise qualitativa com os processos de codificação, categorização e interpretação, do qual resultaram em três categorias: composição da rede, tipo de apoio e o apoio que falta. O apoio predominante foi o instrumental e o emocional, especialmente aquele prestado pela família. As necessidades das pessoas entrevistadas estão relacionadas, dentre outras, à falta de acessibilidade. $\mathrm{O}$ conhecimento das redes de apoio é importante para guiar a prática do profissional enfermeiro e demais membros da equipe de saúde para ampliarem e fortalecerem a interconexão entre os integrantes da rede de apoio.
\end{abstract}

Palavras chave: Pessoas com deficiência, apoio social, enfermagem, reabilitação.

\begin{abstract}
This study focuses on the support network for people with disabilities and how this support is provided. The objective was to identify and understand the support networks and how they support the person with disability. Results of a descriptive qualitative approach, based on semi-structured interviews with 13 people with physical disabilities, six with amputation and seven with spinal cord injury treated at are habilitation center. Qualitative analysis was used with then coding processes, categorization and interpretation, which in three categories: composition of the network, type of support and support which is lacking. The main support was instrumental and emotional, especially that provided by the family. The needs of the interviewed people are related, among others, the lack of accessibility. The knowledge of support net works is important to guide the practice of nurses

\footnotetext{
*Enfermeira, Universidade Federal de Santa Catarina. Florianópolis, Brasil. Email: daniely_m_cruz@hotmail.com

${ }^{* *}$ Enfermeiro, Universidade Federal de Santa Catarina. Florianópolis, Brasil. Email: luisramon.52@gmail.com

${ }^{* * *}$ Enfermeira. Docente do Departamento de Enfermagem da Universidade Federal de Santa Catarina. Florianópolis, Brasil. Email: denise_guerreiro@hotmail.com

${ }^{* * * *}$ Enfermeira. Docente do Departamento de Enfermagem da Universidade Federal de Santa Catarina. Florianópolis, Brasil. Email: soraia.dornelles@ufsc.br
} 
and other members of the healthcare team to expand and streng then the interconnection between the members of the support network.

Key words: People with disabilities, social support, nursing, rehabilitation.

\section{RESUMEN}

Este estudio se centra en la red de apoyo para las personas con discapacidad y cómo se proporciona este soporte. El objetivo fue identificar y comprender la red de apoyo y cómo apoyar a la persona con discapacidad. Se trata de una investigación cualitativa, descriptiva, con entrevistas semiestructuradas de 13 personas con discapacidad física, seis con amputación y siete con lesión medular, atendidas en un centro de rehabilitación. Fue utilizado análisis cualitativo seguiendo la codificación, categorización e interpretación, que resultaron en tres categorías: composición de la red, tipo de apoyo y la ausencia de apoyo. Los apoyos predominantes fueron el instrumental y el emocional, especialmente el procedente de la familia. Las necesidades de las personas entrevistadas están relacionadas, entre otros aspectos, a la falta de accesibilidad. El conocimiento de la red de apoyo es importante para orientar la práctica de las enfermeras y otros miembros del equipo de salud para ampliar y fortalecer la interconexión entre los miembros de la red.

Palabras clave: Personas con discapacidades, apoyo social, enfermería, rehabilitación.

Fecha recepción: 24/09/13 Fecha aceptación: 06/04/15

\section{INTRODUÇÃO}

Este artigo trata de questões relacionadas ao universo das pessoas com deficiência física. Considera que estas pessoas apresentam problemas ortopédicos que interferem em sua movimentação voluntária, impedindo-as total ou parcialmente, de acordo com os padrões de normalidade para os seres humanos (1).

Dados do Instituto Brasileiro de Geografia Estatística, relativos ao Censo Demográfico 2010 (2), mostram que, no Brasil, existem aproximadamente 13 mil pessoas com deficiência física (ou motora). Destas, 11 mil são moradoras de áreas urbanas e 2 mil são habitantes de áreas rurais.

Neste grupo, merecem especial atenção as pessoas com Lesão Medular e aquelas com Amputação de Membros. A Lesão Medular caracteriza-se por uma condição crônica com manifestações incapacitantes. Estudos indicam que cerca de seis a oito mil brasileiros são acometidos por lesão medular anualmente e que a mortalidade em decorrência desta diminui consideravelmente $(3,4)$. Define-se amputação como a retirada, em geral cirúrgica, de um membro, total ou parcialmente. Esta pode ser decorrente de "problemas vasculares, neuropáticos, traumáticos, tumorais, infecciosos, congênitos e iatrogênicos" (5). A maior parte destas ocorre em virtude do crescente número de traumas decorrentes de acidentes automobilísticos (6).

Entretanto, esta parcela da população não é comumente vista nos espaços de circulação coletiva. Esta constatação se deve ao fato de que a sociedade não parece ter a capacidade de atender de maneira integral às necessidades dos indivíduos que a compõem, dificultando a prática da inclusão, da acessibilidade 
e exacerbando o estado de deficiência. Assim, dá vazão à discriminação, preconceito, imposição de barreiras e exclusão sociocultural (7).

O processo de viver com deficiência física não se restringe a dificuldades e limitações. Muitos são os casos de pessoas que transpõem as barreiras encontradas e conseguem viver de acordo com o que se considera normal na sociedade, de forma autônoma e independente. Contudo, é importante que as pessoas com deficiência física mantenham suas relações e estabeleçam novas relações interpessoais, de modo a superar suas dificuldades, sejam elas emocionais ou instrumentais, pois em algum momento podem necessitar do apoio de outras pessoas (4).

A vida da pessoa com deficiência física requer o apoio de diferentes fontes e tipos, o que acaba por promover o desenvolvimento de redes. As redes de apoio ou redes sociais de apoio destacam-se como uma forma de se organizar, atuar, de formar parcerias e alianças, através de um processo dinâmico (8).

De acordo com Sluzki (9), pesquisador referência no estudo das redes sociais no âmbito da saúde: "rede social é a soma de todas as relações que um indivíduo percebe como significativas ou que define como diferenciadas da massa anônima da sociedade (...) e contribui para sua percepção como indivíduo e para sua autoimagem".

A conceituação de redes pode ganhar várias formas, contudo: "mais importante que definir é entendê-la como uma proposta democrática de realização do trabalho coletivo e de circulação do fluxo de informações, elementos essenciais para o processo cotidiano de transformação social", é o que destaca Meirelles (10). Segundo Sluzki (9) o apoio prestado pelas redes pode se estabelecer de diferentes formas, dentre as quais figuram: $o$ apoio informacional, que consiste no compartilhamento de informações, esclarecimento de expectativas, orientações, aconselhamentos, entre outros; o apoio emocional, aquele estabelecido nas relações familiares e de amizade, envolvendo simpatia, empatia, estímulo e auxílio; e o apoio instrumental, configurado como "ajuda material e de serviços", propondo-se a sanar as necessidades de materiais em geral e proporcionar ajuda para a realização de trabalhos práticos da vida.

Considerando a relevância do apoio necessário as pessoas com deficiência física e a lacuna que existe no conhecimento sobre quem integra esta rede, que tipo de apoio elas fornecem, surgiu a questão que norteou este estudo: Quais são as redes de apoio social das pessoas com deficiência física? O objetivo do estudo foi conhecer as redes de apoio social à pessoa com deficiência física, residentes da Grande Florianópolis.

\section{MATERIAL E MÉTODO}

Trata-se de pesquisa descritiva com abordagem qualitativa, realizado durante os meses de abril a junho de 2013, em um centro de reabilitação referência no Estado de Santa Catarina, Brasil.

A pesquisa qualitativa de acordo com Minayo (11) se aplica ao estudo da história, das relações, das representações, das crenças, das percepções que os humanos descrevem de como vivem e sobre si mesmos, como pensam e sentem. A autora afirma que a pesquisa se dá a partir da interpretação dos fatos pelo sujeito conforme seu cotidiano, levando em consideração o que o sujeito pensa sobre o que está sendo investigado.

A partir da obtenção da relação das pessoas que poderiam atender aos critérios de inclusão fornecida por este centro de referência em reabilitação, as pessoas foram contatadas e as entrevistas realizadas no domicilio, escola profissional, universidades, centro comercial, hospital e centro de reabilitação.

Os participantes do estudo foram treze pessoas com deficiência física, especialmente 
com lesão medular e amputação, com idade entre 18 a 60 anos, de ambos os sexos, residentes nos municípios de Florianópolis, São José e Palhoça, do estado de Santa Catarina. Foram excluídas da pesquisa as pessoas que além da deficiência física apresentaram outro tipo de deficiência, como deficiência cognitiva ou intelectual.

A amostra foi intencional, a escolha das pessoas com lesão medular e amputação ocorreu devido à pouca produção científica e estudos relacionados à temática da deficiência física e rede de apoio, e ao expressivo número de casos no Brasil nos últimos anos.

A coleta de dados foi realizada através de entrevistas semiestruturadas, onde inicialmente foram realizadas perguntas em relação aos aspectos sociodemográficos e da condição de saúde-doença. Após, foi perguntado o que havia mudado em suas vidas após a deficiência e que ajuda haviam recebido e ainda recebiam, explorando esse aspecto de forma a conhecer a rede de apoio dessas pessoas.

Destacamos que as informações passaram a se repetir a partir da décima primeira entrevista, não havendo informações novas acerca de quem eram os integrantes da rede, que tipo de apoio esta rede fornecia e as necessidades de apoio que ainda sentiam falta. Esta percepção nos orientou na delimitação do número de participantes.

A análise das entrevistas das pessoas com deficiência física foi realizada através da codificação dos dados, categorização, descrição e interpretação dos mesmos. A categorização foi realizada considerando: quem ajudou/ajuda; tipo de ajuda recebida; e o que sentiram falta.

Os aspectos éticos de pesquisa em seres humanos foram preservados seguindo-se a preconização das normas de pesquisa em saúde expressa na Resolução No 196, de 10 de outubro de 1996, do Conselho Nacional de Saúde (12). O projeto de pesquisa foi enviado para apreciação ao Comitê de ética e Pesquisa da Universidade Federal de Santa Catarina aprovado pelo parecer de número 216.396 de 11 de março de 2013.

Tendo em vista a garantia do anonimato, os entrevistados estão identificados com siglas de acordo com a deficiência, LM para pessoas com lesão medular, e AMP para pessoas com amputação, seguido do número que corresponde à entrevista.

\section{RESULTADOS}

O Tabela 1 apresenta algumas características dos participantes do estudo, com seis pessoas com amputação e sete com lesão medular. Todos eram adultos, com idade variando de 18 a 60 anos, com nível de escolaridade variável entre ensino fundamental incompleto e ensino superior incompleto. No que diz respeito ao estado civil, oito eram solteiros, quatro casados e um era separado. Quanto à religião figuraram o catolicismo citado por sete entrevistados, a religião evangélica mencionada por três entrevistados, um era espírita e dois que não professavam religião alguma. A renda mensal variou entre um a sete salários mínimos.

As causas das deficiências foram variadas, porém houve um destaque para o acidente de moto como causa mais comum, especialmente das amputações.

A rede de apoio dessas pessoas será apresentada nas três categorias, que seguem: 
Tabela 1. Características dos participantes do estudo.

\begin{tabular}{|c|c|c|c|c|c|c|}
\hline $\begin{array}{c}N^{\circ} \\
\text { Entrev. }\end{array}$ & Sexo & Idade & $\begin{array}{l}\text { Tempo de } \\
\text { Deficiência }\end{array}$ & Tipo de Deficiência & Causa & $\begin{array}{c}\text { Tipo de equipamento } \\
\text { que usa }\end{array}$ \\
\hline AMP 1 & $\mathrm{~F}$ & 52 & $16 \mathrm{a}$ e $2 \mathrm{~m}$ & Amp. Transradial D & Acidente de moto & - \\
\hline AMP 2 & $\mathrm{~F}$ & 18 & la e $9 \mathrm{~m}$ & Amp. Transfemural E & Acidente de moto & Muletas \\
\hline AMP 3 & M & 31 & $12 \mathrm{a}$ & Amp. Pé E e $5^{\circ}$ Dedo da mãe E & Acidente de moto & Prótese. \\
\hline AMP 4 & $\mathrm{~F}$ & 60 & $13 a$ & Amp. Transfemural E & Acidente de moto & Prótese \\
\hline AMP 5 & $\mathrm{~F}$ & 59 & $28 \mathrm{a}$ & Amp. Transfemural & Acidente de moto & Prótese \\
\hline AMP 6 & M & 40 & $9 \mathrm{~m}$ & Amp. Transfemural E & Acidente de moto & Cadeira de rodas e muletas \\
\hline LM 1 & $\mathrm{~F}$ & 30 & 4a e $10 \mathrm{~m}$ & Lesão medular & Esclerose múltipla & - \\
\hline LM 2 & M & 43 & 3 a e $5 \mathrm{~m}$ & Lesão medular & Mergulho em águas rasas - mar & Cadeira de rodas \\
\hline LM 3 & $\mathrm{~F}$ & 37 & $5 \mathrm{a}$ & Lesão medular & Anestesia durante cesariana & Cadeira de rodas \\
\hline LM 4 & M & 33 & $2 \mathrm{a}$ e $6 \mathrm{~m}$ & Lesão medular & Acidente de moto & Cadeira de rodas \\
\hline LM 5 & M & 28 & $4 a \mathrm{e} 2 \mathrm{~m}$ & Lesão medular & Ferimento por arma de fogo & Cadeira de rodas \\
\hline LM 6 & M & 22 & $5 \mathrm{a}$ e $10 \mathrm{~m}$ & Lesão medular em C6/C7 & Acidente de moto & Cadeira de rodas \\
\hline LM 7 & M & 35 & $12 \mathrm{a} e 3 \mathrm{~m}$ & Lesão medular em C5/C6 & Acidente de moto & Cadeira de rodas e muletas \\
\hline
\end{tabular}

\section{Composição da rede}

De acordo com os achados em relação à composição da rede, a família se caracterizou como principal fonte de apoio. Dentro da família pais, irmãos e maridos foram considerados importantes fonte de ajuda, seguidos de tios, sogros, genros, namorados e sobrinhos. A figura da mãe surgiu como o componente familiar mais referenciado, mostrando predomínio do apoio feminino, porém, também com a presença da figura masculina. A relevância do apoio familiar é evidenciada na fala que segue:

Eu tive sorte de ter a minha família que me apoiou muito, a minha mãe na época tava construindo né, na época que eu fiquei na cadeira de rodas ela estava construindo. Ela construiu a casa toda adaptada, pensando no depois né. Ela dizia assim, minha filha não vai ficar na cadeira de rodas, mas se um dia isso acontecer eu quero que ela seja muito bem vinda na minha casa. (LM1)
Amigos foram consideravelmente citados como apoio, assim como vizinhos e pessoas desconhecidas. Também foram citados na pesquisa grupos e organizações, que incluíam as redes sociais (internet), mídia televisiva, pessoas com problemas semelhantes, grupos religiosos, motoristas de ônibus e equipes esportivas. O apoio recebido por meio das relações de trabalho foi referenciado por apenas dois dos entrevistados.

Dentre os serviços de saúde citados como apoio destacam-se centros de reabilitação, hospitais públicos e privados, clínicas privadas e centros de saúde na atenção primária. Nestes serviços foram citados em maior número os profissionais médicos, seguidos por fisioterapeutas, nutricionista, enfermeiros e outros profissionais de enfermagem.

[...] tenho acompanhamento com nutricionista daqui mesmo. Fisioterapia eu dei uma parada por causa da bacia. E venho na Enfermagem por causa de problemas tipo remédio, às vezes sai um machucado, a gente tem que estar cui- 
dando, tratando para não virar uma escara tal. [...] Quem mais me apoiou foram as enfermeiras daqui. Os fisioterapeutas que também me apoiaram bastante. [...] eu sou quem eu sou porque eles me ajudaram depois da paraplegia. (LM5)

Houve uma variedade de instituições citadas, dentre elas, prefeituras, Centro de Referência de Assistência Social (CRAS), universidades públicas e privadas, associações de classe e escritório de advocacia.

\section{Tipo de apoio}

Com relação aos tipos de apoio observou-se predomínio do apoio instrumental. Este caracterizou-se por meio de: auxílio financeiro evidenciado pela doação de dinheiro; compra de materiais de consumo (alimentos, medicamentos) e de uso permanentes (equipamentos ortopédicos e materiais de construção); realização de eventos para angariar fundos; e recebimento de auxílios previdenciários em forma de pensão e aposentadoria. Também podemos destacar o auxílio nas atividades do cotidiano, tais como: auxílio na realização de afazeres domésticos; transportes; locomoção; e atividades de lazer.

Ainda com relação ao apoio instrumental, foram destacados os cuidados das pessoas com deficiência física que se caracterizaram por: cuidados de higiene e conforto; realização de procedimentos como, curativos, sondagem vesical, aferição de pressão arterial; e o acompanhamento na internação hospitalar. Em relação ao seu tratamento houve destaque ao processo de reabilitação, no qual as especialidades citadas foram: fisioterapia; ortopedia; neurologia; nutrição; e psicologia. Além das atividades desportivas, como natação, rugby em cadeira de rodas e musculação.

Entre os deficientes físicos entrevistados houve um predomínio do auxílio financeiro, voltado para o tratamento, compra de remédios e equipamentos, como pode-se evidenciar na fala a seguir:
Meus amigos fizeram ali alguns eventos pra realmente angariar fundos para o meu tratamento. Eu particularmente fiz alguns eventos né, convidando amigos pra tocarem, pra divulgarem, pra me auxiliar aí com a questão da aparelhagem de som, instrumentos, o transporte das pessoas, a questão da segurança do evento, portaria e tal, foram todos amigos assim né. (AM 2)

No que se refere ao apoio emocional os entrevistados referenciaram, principalmente a família que presta este apoio através de visitas, conversas, companhia, demonstrações de afeto, contatos telefônicos ou por mensagens. Os entrevistados entendem esse apoio como forma de não entrar em depressão, de superar as dificuldades e tornar-se autônomo e independente, de compartilhar os problemas, de transformação pessoal tornando-se "o que são hoje", apoio moral e psicológico, elucidados na seguinte fala:

Então assim é um apoio fundamental para qualquer recuperação, e eu acredito que a minha recuperação só foi possivvel, com tudo isso! É olhar para eles e dizer assim... Eu tenho que ser mais forte não só por mim, mas por eles, por que eles confiam, eles confiam que eu vou melhorar. (LM 1)

O apoio informacional não foi destacado por todos os entrevistados da pesquisa. Porém, este apareceu através do compartilhamento de informação sobre o viver com a prótese, ensinar a viver melhor; orientações de adaptação no ambiente domiciliar, orientação para a realização de procedimentos invasivos, como autocateterismo. Além disso, destacaram os encaminhamentos para especialidades e serviços de saúde, retiradas de dúvidas sobre o tratamento e situação de saúde e apoio jurídico.

Eu, quem me ensinou a passar a sonda sozinha foi a enfermeira lá na reabilitação, eu tinha dificuldade de ir ao banheiro, eu colocava espelho, 
eu colocava tudo e nada dava certo, mas por que eu não estava na posição correta. Um dia ela falou você vem aqui que eu vou te ensinar. Ela me colocou na maca, ai "oh assim, fica nessa posição, tá enxergando?! Coloca o espelho, ai agora vai", e foi a partir daquele dia foi, mas precisou daquilo ali. (LM 1)

\section{O apoio que faltou}

A maior carência citada pelas pessoas com deficiência física estava relacionada à falta de acessibilidade aos ambientes de circulação pública, estabelecimentos comerciais e outros serviços. Dentre estes, se destacaram: repartições públicas de difícil acesso; falta de acessibilidade nas ruas, por meio de calçadas inadequadas; faltas de rampas ou rampas de angulação inapropriada; escadas com degraus irregulares ou em grande quantidade que dificultam o acesso. Também foram citadas a falta de conscientização das pessoas sobre a realidade das necessidades das pessoas com deficiência física e a falta de educação no trânsito. A falta de acessibilidade evidencia-se na fala a seguir:

Não só eu, mas qualquer portador de deficiência física, mental, visual, a maior barreira que encontra é a acessibilidade. Calçadas, falta de rampas, negligência das pessoas que não tem deficiência fisica. E acabam atrapalhando os poucos acessos que o portador de deficiência física tem. Atrapalhando, se colocando onde não é para estar, no caso de estacionamento. É isso aí, a maior carência é a acessibilidade. (LM7)

Em relação aos seus cuidados e tratamentos as pessoas entrevistadas destacaram como um apoio a ser alcançado, a busca por mais atendimentos nas especialidades de fisioterapia, psicologia, educação física, nutrição, terapia ocupacional e atendimento médico domiciliar e nos Centros de Saúde. Alguns entrevistados destacaram também a necessidade de contratação de mais profissionais nos serviços de saúde, assim como melhorias na estrutura física destes espaços, obtenção de próteses de boa qualidade, equipamentos, medicação e material para tratamentos.

Em tudo, eu achava que a presidente deveria ajudar o deficiente dando uma prótese melhor. Porque se eu tenho uma prótese boa, eu posso fazer tudo sem depender de ninguém. [...] Depois que acabou a garantia começam a aparecer os defeitos todos. Então isso aí para mim não é garantia. Garantia é para ter revisão para o resto da vida da gente. Essa minha aqui deve ter quase oito anos, enquanto que a nova está lá no canto, porque não presta. É um material que não presta. É só isso que eu reclamo. Porque uma perna mecânica é cara. (AM5)

Com menor destaque foram citadas outras necessidades como voltar a estudar, a trabalhar e a dirigir. Apenas um entrevistado mencionou não sentir falta de apoio, isso se deu, possivelmente, pelo menor nível de comprometimento de sua deficiência.

\section{DISCUSSÃO E CONCLUSÕES}

De acordo com o que se evidenciou nos resultados desta pesquisa, o apoio prestado pela família destaca-se em relação aos demais. Mostra-nos a literatura que tal evidencia deve-se ao fato de que a pessoa que vivencia uma condição crônica, como a deficiência física, requer atenção constante ao menos em alguma fase de sua vida, incumbindo à família, principalmente a nuclear, de suprir as demandas de seu cuidado (13). Apesar desta constatação, muitas vezes, a ocorrência da deficiência na família pode causar situações de crise no ambiente familiar, abalando sua estrutura e funcionamento, quando esta se vê despreparada para lidar com a nova condição de seu ente. É necessário que a família adapte-se à realidade imposta, tentando se reorganizar para a vida com a deficiência, a fim de reconstituir sua identidade como gru- 
po familiar (14).

A família ocupa-se das preocupações relacionadas ao cuidado da pessoa com deficiência física, quanto à manutenção do conforto, higiene, alimentação, adaptação a nova condição. Além destes, os membros da família preocupam-se em relacionar estes cuidados com outras responsabilidades da vida e preocupam-se com o futuro daqueles a quem prestam apoio (15).

Apesar de termos evidenciado expressiva existência do apoio prestado por figuras masculinas da família, houve o predomínio do apoio feminino oriundo de mães, irmãs, namorada e tias. Esta constatação se dá, possivelmente, ao fato de que as mulheres parecem apresentar maior desenvoltura no desenvolvimento de relações interpessoais, sendo estas mais íntimas e duradouras. Culturalmente, a figura feminina exerce participação marcante no cuidado, soluciona problemas, busca informações, favorecendo as relações de apoio. Os homens apoiam no deslocamento das pessoas com deficiência física realizam compras, marcam consultas com especialistas $(14,15)$. A referência ao apoio prestado pelas mulheres deve-se também ao fato de que a maioria dos participantes deste estudo foram homens e jovens.

Contudo, de acordo com os resultados, não é apenas o apoio prestado pela família que se destacou. Evidenciou-se o apoio advindo das relações de amizade, de vizinhos, de grupos, organizações e das relações de trabalho, que se interligam e complementam o apoio familial. Estes, no âmbito das relações mais íntimas, desenvolvem o papel de cuidadores informais, dando suporte emocional e amparo para que a pessoa com deficiência se beneficie, sendo mais autônoma e independente (13).

As pessoas com deficiência física necessitam de cuidados de saúde e acompanhamento especializado ao longo de toda a sua vida e estes são importantes fontes de apoio. Neste sentido merece destaque a reabilitação físico-motora que exerce importante papel no processo saúde-doença das pessoas com deficiência física e exigindo atuação de uma equipe interdisciplinar que se interligam e cooperam para a atenção dos objetivos do cuidado (16). Outros serviços de saúde destacam-se, como hospitais e clínicas privadas, surgem como complemento ao apoio em saúde. $\mathrm{O}$ apoio prestado por instituições como universidades, instituições de classe, entidades governamentais são menos expressivos na rede de apoio, contudo, podem se caracterizar como importantes fontes de apoio emocional e material (13).

O cotidiano da pessoa com deficiência física inter-relaciona diversas formas de apoio que buscam sanar as necessidades decorrentes das mudanças e limitações do viver com deficiência física, constituindo sua rede de apoio social.

O apoio emocional de acordo com Sluzki (9) é um processo de ajuda através de atitude emocional positiva, reforçando a estima e a confiança dos sujeitos. Os deficientes físicos referenciam principalmente a família, dentre eles destacando a mãe e o parceiro como fonte desse apoio emocional. Para Tavares e Silva (8) em estudo sobre a rede de apoio social de pessoas com hipertensão arterial, a família representa importante fonte de apoio e segurança, permite trocas de amor, afeto, respeito e valor, influenciando diretamente no sucesso do tratamento de pessoas com doenças crônicas.

De acordo com Souza (17) o apoio emocional se refere a disponibilidade de alguém com quem conversar e inclui as condutas que buscam sentimentos de bem-estar afetivo. Demonstrações essas que se expressam principalmente na garantia de dar o melhor, de estar presente e de evidenciar sua preocupação.

Sobre o apoio instrumental Sluzki (9) afirma que está relacionado ao auxílio material ou de serviços, de outras atividades, até auxílio financeiro ou material. Semelhante ao que encontramos no estudo de Tavares e Silva (8), realizado com pacientes hipertensos, 
identificou que o apoio instrumental pode ser espontâneo em alguns casos, mas ainda pode aparecer somente quando solicitado conforme as necessidades da pessoa com doença crônica. Tais necessidades podem ser de caráter financeiro; de caráter operacional como levar e acompanhar em exames e consultas no serviço de saúde, por exemplo. Aqueles relacionados aos serviços de saúde são prestados pelos profissionais, tais como prover as consultas médicas e de enfermagem, bem como providenciar exames clínicos e remédios (8).

Maciel (18) refere que a família é promotora de ajuda material e de serviço em necessidades do cotidiano, não apenas com cuidado a doença crônica. Em relação ao apoio vinculado aos serviços de saúde, Tavares e Silva (8) relatou que provém dos atendimentos das demandas das pessoas com doenças crônicas, como disponibilidade de consulta médica, prevenção e controle de complicações, atendimentos nas unidades de saúde como em serviços especializados.

O apoio informacional, segundo Sluzki (9) refere-se ao compartilhamento das informações, esclarecimento de dúvidas, oferecimento de conselhos e orientações que possam solucionar seus problemas e adquirir conhecimentos.

Este tipo de apoio aparece quando solicitado a alguém pela própria pessoa ou quando elas buscam informação a respeito da doença, do tratamento e assuntos relacionados. Neste caso, as fontes de informação incluem mídia escrita e falada; vizinhos e familiares, dentre outros (8).

A rede de apoio social, segundo Tavares e Silva (8) permite ou pelo menos tem a intenção de permitir às pessoas um viver melhor, mesmo na presença de doenças crônicas. No atendimento às principais demandas geradas pelo surgimento da doença, a rede de apoio social mostra suas ações, é valorizada e reconhecida. $\mathrm{Na}$ avaliação que as pessoas fazem do apoio, essas questões são destacadas, entretanto as lacunas são tam- bém percebidas (8).

A falta de acessibilidade aos ambientes de livre circulação das pessoas, fator marcante nos achados da pesquisa, posto que foi citada pela maioria dos entrevistados, causa nas pessoas com deficiência física um sentimento de viver presas, encarceradas, privadas de realizar suas necessidades básicas (19). Tal lacuna vai de encontro ao que versa o Artigo $5^{\circ}$ da Constituição Federal do Brasil, que diz que "é livre a locomoção no território nacional em tempo de paz, podendo qualquer pessoa, nos termos da lei, nele entrar, permanecer ou dele sair com seus bens" (20).

Essa falta de apoio se dá pelo fato de que a rede de apoio social dos deficientes físicos não pode atender a todas as demandas provenientes do viver com a deficiência física, não podendo, portanto, intervir e solucionar os problemas.

A legislação brasileira garante à pessoa com deficiência física uma série de direitos, que preconizam desde a promoção da acessibilidade à benefícios previdenciários. Contudo, somente a existência de tais direitos parece não bastar para promover a integração dessas pessoas. São necessárias, além da sensibilização da sociedade acerca da temática deficiência física, ações que primem pelo que lhes é garantido legalmente.

No entorno da pessoa com deficiência física desenvolve-se uma teia de inter-relações denominada rede de apoio, que tem a finalidade de suprir suas necessidades. O apoio mais significativo prestado a pessoa com deficiência física é aquele vinculado a família, onde a família nuclear se destaca. Esta se ocupa desde os cuidados básicos do cotidiano até as preocupações relacionadas ao futuro dessas pessoas. Outras fontes de apoio são significativas, como relações de amizade, trabalho e de profissionais de saúde. Estes se interligam e se complementam com o apoio prestado pela família. Prestam apoio relacionado a ajuda financeira e material, apoio emocional e disponibilizam informações para o enfrentamento da vida com deficiência. 
O conhecimento das redes de apoio à pessoa com deficiência física possibilita ao profissional enfermeiro e aos demais membros da equipe de saúde compreender as condições, as limitações e as possibilidades de quem vive e convive com uma deficiência física. Além disso, possibilita a relação com os componentes da rede com vistas ao aprimoramento da atenção prestadas a pessoa com deficiência física.

O enfermeiro é membro da equipe de reabilitação, contudo a deficiência física é pouco ou nunca abordada nos campos de formação e produção de conhecimento. Evidencia-se assim a necessidade de maior preparo daqueles que serão os profissionais a atender as pessoas com deficiência física em algum momento de sua vida. Esperamos que esse estudo dê subsídios para a formação e a prática da enfermagem frente a estas pessoas.

\section{REFERÊNCIAS}

1. Organização Mundial de Saúde. Relatório mundial sobre a deficiência/World Health Organization, The World Bank; tradução Lexicus Serviços Linguísticos. São Paulo: SEDPcD, 2012. 334 p.

2. Instituto Brasileiro de Geografia Estatística. Censo Demográfico 2010. Características gerais da população, religião e pessoas com deficiência. Rio de Janeiro; 2010.

3. Ministério da Saúde do Brasil; Secretaria de Atenção à Saúde, Departamento de Ações Programáticas Estratégicas, Departamento de Atenção Especializada. Diretrizes de Atenção à Pessoa com Lesão Medular. Brasília: Ministério da Saúde; 2012.

4. Fechio MB, Pacheco KMB, Kaihami HM, Alves, VLR. A repercussão da lesão medular na identidade do sujeito. Acta Fisiátr. 2009; 16 (1): 38-42.

5. Silva EFSJ, Flores ACF, Folmer V, Brito VB.
Perfil de 52 usuários amputados atendidos no Hospital Santa Casa de Caridade de Uruguaiana. In: Salão Internacional de Ensino, Pesquisa e Extensão. Bagé: SIEPE; 2009.

6. Senefonte FRA, Rosa GRPS, Comparin ML, Coure MR, Jafar MB, Andrade FAM, et al. Amputação primária no trauma: perfil de um hospital da região centro-oeste do Brasil. J Vasc Bras 2012; 11 (4): 269-276.

7. Reboucas CBA, Cezario KG, Oliveria PMP, Pagliuca LMF. Pessoa com deficiência física e sensorial: percepção de alunos da graduação em enfermagem. Acta Paul Enferm. 2011; 24 (1): 80-86.

8. Tavares RS, Silva DMV. A implicação do apoio social no viver de pessoas com hipertensão arterial. Rev Gaucha Enferm. 2013; 34 (3): 14-21.

9. Sluzki, C.E. A rede social na prática sistêmica. São Paulo: Casa do Psicólogo, 1997.

10. Meirelles BHS. Redes sociais em saúde: desafio para uma nova prática em saúde e Enfermagem. [Trabalho apresentado para Concurso Público ao cargo de Professor Adjunto] Florianópolis: Universidade Federal de Santa Catarina; 2004.

11. Minayo MCS. Pesquisa Social: Teoria, método e criatividade. 29a ed. Rio de Janeiro: Vozes; 2010.

12. Ministério da Saúde do Brasil; Conselho Nacional de Saúde, Comissão Nacional de Ética em Pesquisa. Normas para pesquisa envolvendo seres humanos - Res. CNS N 196/96 e outras. Brasília: Ministério da Saúde; 2003.

13. Rodrigues JSM, Ferreira NMLA. Estrutura e funcionalidade da rede de apoio social do adulto com câncer. Acta Paul Enferm. 2012; 25 (5): 781-787.

14. Barbosa MAM, Balieiro MMFG, Pettengill MAM. Cuidado centrado na família no contexto da criança com deficiência e sua família: uma análise reflexiva. Texto contexto - enferm. 2012; 21 (1): 194-199.

15. Sánchez KOL, Ferreira NMLA, Dupas G, 
Costa DB. Apoio social à família do paciente com câncer: identificando caminhos e direções. Rev Bras Enferm. 2010; 63 (2): 290-299.

16. Alvarado A, Moreno ME, Rodríguez MC. Inclusión social y participación comunitaria: una perspectiva de trabajo frente a la discapacidad. Cienc. enferm. 2009; XV(1): 61-74.

17. Souza, SS. Apoio às pessoas com tuberculose e redes sociais [Tese de Doutorado]. Florianópolis: Universidade Federal de Santa Catarina, Programa de Pós-Gradu- ação em Enfermagem; 2010.

18. Maciel KF, Ulbrich EM, Labronici LM, Maftum MA, Mantovani MF, Mazza VA. A hipertensão arterial na percepção dos seus portadores. Cienc Cuid Saude. 2011; 10(3): 437-443.

19. Nascimento, VF. Acessibilidade de deficientes físicos em uma unidade de Saúde da Família. Gestão e Saúde. 2012; 3 (3): 1031-1044.

20. Brazil. Constituição da República Federativa do Brasil; 1988. 